\title{
Morpho-Physical and Chemical Characteristics of Mountain Soils in Central Leyte
}

\author{
Deejay S. Maranguit and Victor B. Asio
}

Department of Agronomy and Soil Science, Visayas State University, Baybay City, Leyte 6521-A, Philippines

\begin{abstract}
An important prerequisite to sustainable soil management is a good understanding of soil morphological, physical and chemical characteristics. Until now, very limited data are available on the characteristics of mountain soils in the central part of Leyte Island. Thus, this study was conducted to determine the morpho-physical and chemical characteristics of mountain soils derived from andesite and shale in central Leyte, and to evaluate the effects of parent material and topographic position on the properties of the soils.

Eight soil profiles representing the dominant parent materials and topographic positions in the Abuyog-Mahaplag-Baybay portion of the central mountain range of the island were examined. Results revealed that the soils varied in their morphological, physical and chemical characteristics which to some extent reflect the nature of their parent material and the slope positions where they were formed. Soils derived from shale (Soil profiles 1, 2, 3 and 6) developed into young (Fluventric Eutropepts) and well-developed (Typic Hapludalfs) soils with generally moderate nutrient status. Soils derived from andesite (Soil profiles 4, 5, 7 and 8) developed into young (Typic Dystropepts) and well-developed (Typic Kandiudults) soils with generally low nutrient status. Regardless of parent material, soils on summit slope position tended to be better developed than soils on footslope and shoulder slope positions. The study showed that the influence of parent material on soil development was modified by the topographic position.
\end{abstract}

Keywords: volcanic soil, sedimentary soil, Leyte central highlands, soil characteristics

Correspondence: D. S. Maranguit Address: Department of Agronomy and Soil Science, Visayas State University, Baybay City, Leyte 6521-A, Philippines. Tel: +63-053-335-2627. Email: dmaranguit@yahoo.com

DOI: $10.32945 /$ atr3514.2013 


\section{INTRODUCTION}

Soil is a vital natural resource since it provides anchorage, nutrients, water, and air to plants. In the Philippines, large areas of lands are degraded annually due to unsuitable land uses and improper soil management practices (Asio et al., 2009). A prerequisite to effective soil protection efforts is a good understanding of soil morphological, physical and chemical characteristics so that proper management such as tillage operation, choice of crops, lime and fertilizer application, irrigation, drainage, and other practices can be done.

Previous pedological studies conducted in some parts of Leyte Island revealed the crucial role of parent material, topographic or landscape position, and land use in the development of soils from sedimentary and volcanic rocks (Asio, 1996; Zikeli et al., 1999; Navarrete et al., 2008). Barerra et al. (1954) grouped the soils of Leyte into poorly-drained flat lowlands, moderately-drained flat lowlands, well-drained flat lowlands, and well-drained rolling uplands. Zikeli et al., (1999) and Navarrete et al. (2008) found that Andisols formed Quaternary volcanic deposits in the central highlands of Leyte. They concluded that high degree of desilification and loss of basic elements mark the final stage for the Andisol. Asio et al. (2006) reported differences in soil characteristics in different topographic positions of a limestone hill in Baybay, Leyte. They found that the soils on the upper slopes (summit, shoulder, and upper backslope) had thin solum, black surface horizon, clayey texture, granular structure, high contents of $\mathrm{OM}, \mathrm{N}, \mathrm{Ca}$ and $\mathrm{CaCO}_{3}$, low $\mathrm{P}, \mathrm{K}, \mathrm{Fe}, \mathrm{Mn}$, and $\mathrm{B}$ contents, and neutral to alkaline pH values. According to Ruhe (1960), the main features of the topographic positions (or landscape positions) are as follows: summit (flat and most stable position), shoulder (convex and surface runoff is at maximum), backslope (dominant process is transport), footslope (concave and depositional surface), and toeslope (constructional surface).

Navarrete et al. (2011) observed a strong influence of external material contribution particularly volcanic ash deposition on the progression of soils from Quaternary sedimentary rocks in Leyte. They concluded that the most important pedogenic processes that formed the soils seem to be weathering and clay formation, loss of bases and acidification, inorganic $\mathrm{C}$ accumulation, structure formation, desilication, and ferrugination. The characteristics of the parent material and topography have controlling influence on the development of the soils. 
The studies cited above demonstrate the major influence of parent material and topography on the formation of Leyte soils. These also clearly point to the fact that the fertility and constraints of the soils are closely linked to their genesis as has been reported by Sanchez (1976) and Hartemink and Bridges (1995). Until now, no detailed information is yet available on the mountain soils in the Abuyog-Mahaplag-Baybay portion of the central highlands of Leyte. In fact, even the Soil Survey of Leyte (Barrera et al., 1954) does not include a description of the soils in this mountain region. Thus, this study was conducted to determine the morpho-physical and chemical characteristics of mountain soils derived from andesite and shale in the central part of Leyte and to evaluate the effects of parent material and topographic position on the properties of the soils.

\section{MATERIALS AND METHODS}

\section{The Study Area}

The study was conducted in the central mountain range of Leyte extending from Abuyog in the eastern side to Mahaplag in the middle portion to Baybay in the western side of the range (Figure 1). Detailed site characteristics of the soils studied are presented in Table 1 . The study site is characteristically rugged and mountainous with elevation ranging from 45 to $357 \mathrm{~m}$ above sea level. The geomorphology of the area is closely related to the formation of Leyte Island which was brought about by tectonic movement and plate convergence in the Tertiary and Quaternary (JICA, 1990; Aurelio, 2000). Uplift and block faulting and volcanism due to the left-lateral strike-slip Philippine Fault that traverses the central part of the island explain the widespread occurrence of volcanic rocks, except in the northwest and southeast of the islands where exposed sedimentary rocks are widespread because they are part of the Visayan Basin (JICA, 1990; Aurelio, 2000; Dimalanta et al., 2006). Mean annual rainfall of Leyte is about $2800 \mathrm{~mm}$ while the mean annual temperature is $28^{\circ} \mathrm{C}$ which can be classified as a tropical rain forest climate (Af) in Köppen's climate classification (Asio, 1996).

Figure 2 shows the dominant parent rocks in the study area which are andesite and shale. Andesite is an intermediate volcanic rock containing 53-65 percent silica. Its most important mineral components are plagioclase feldspar, amphiboles, pyroxenes, biotite and quartz. Shale is a laminated and thinly bedded fine-grained clastic sedimentary rock 
containing mainly silt and clay and including variable amounts of quartz grains and its typical color is gray (Huang, 1962).

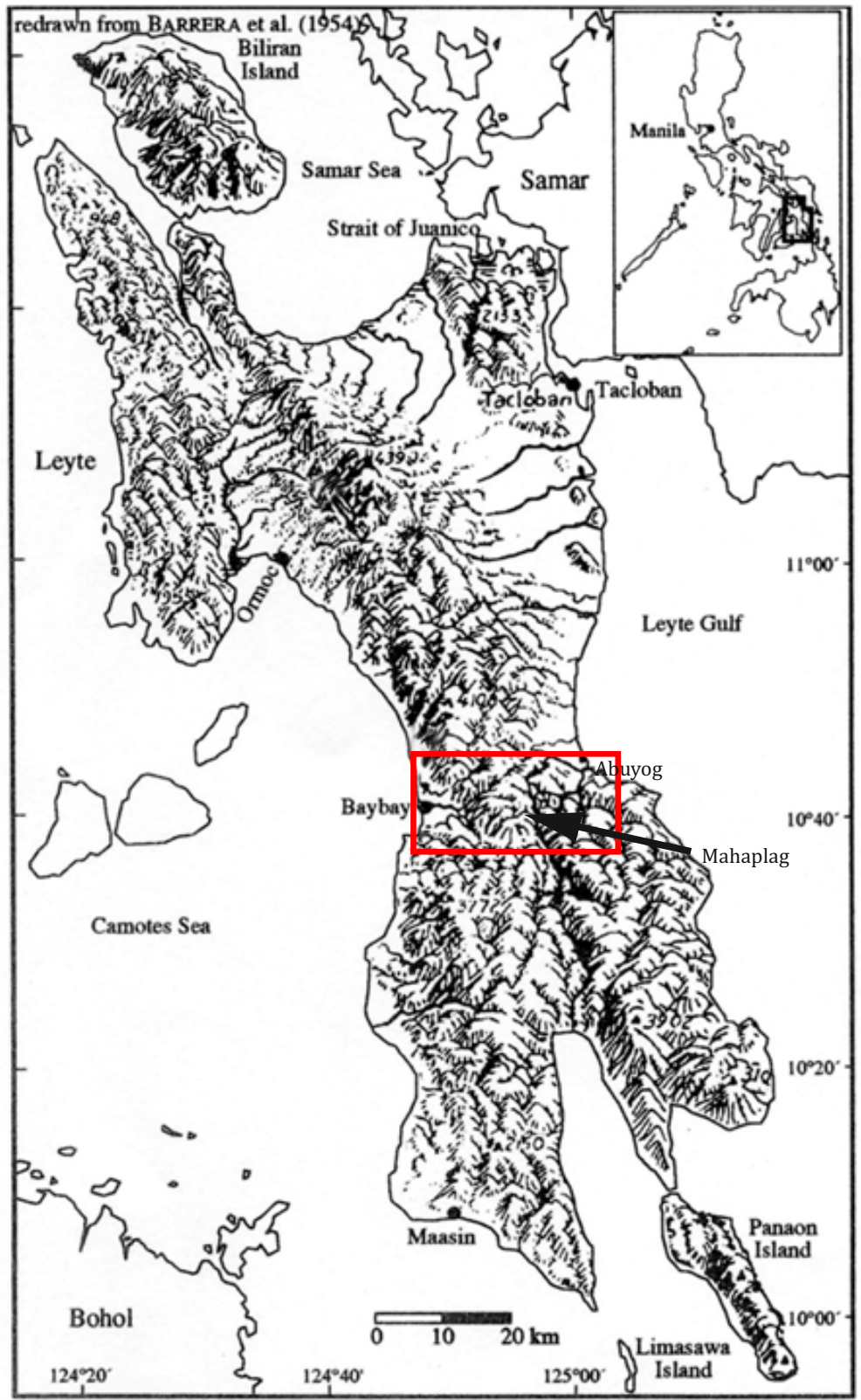

Figure 1. Location of the study site in central Leyte 


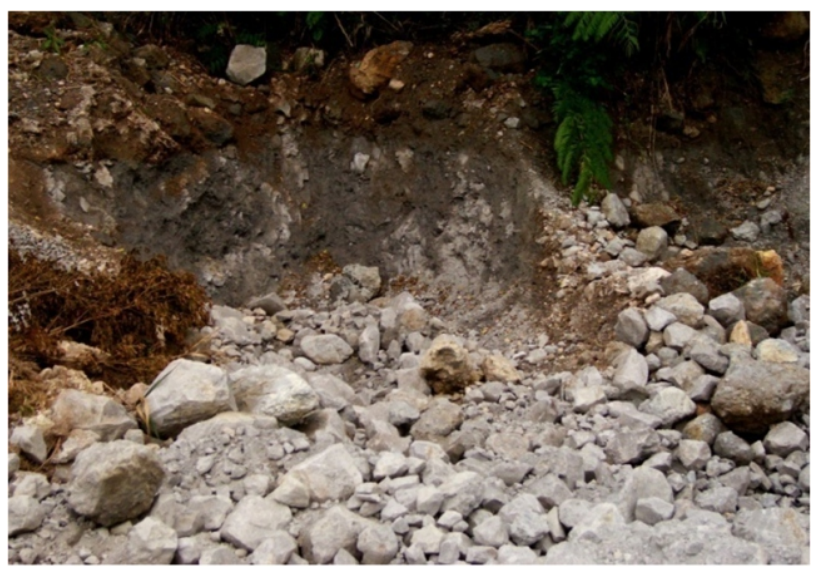

Andesite

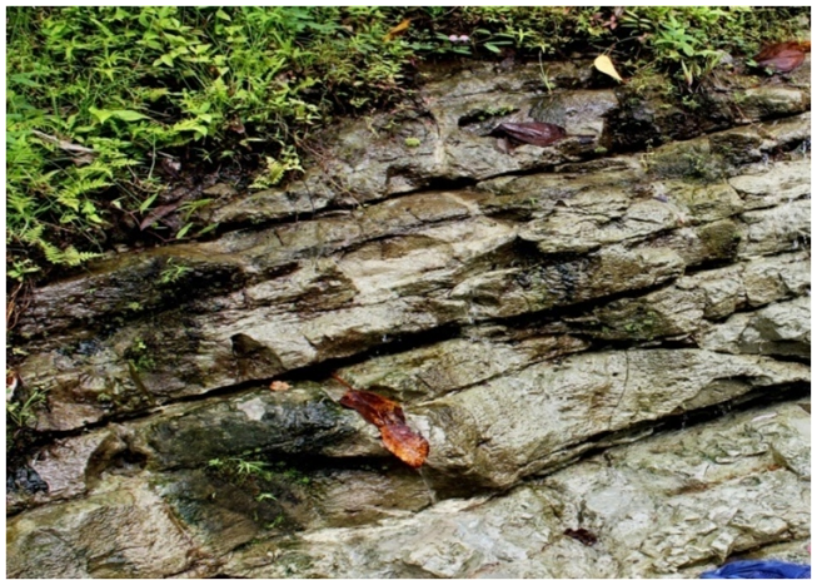

Shale

Figure 2. The andesite and shale parent rocks of the soils in the study area

\section{Soil Profile Characterization, Sampling and Preparation}

A pit measuring approximately $1 \mathrm{~m} \times 1 \mathrm{~m}$ and having a depth of at least $1 \mathrm{~m}$ was dug manually in each site to examine and sample the soil profile. Alternatively, road cuts were cleared by removing about 20 to $30 \mathrm{~cm}$ thickness of surface soil to expose the fresh soil. Site and soil profile descriptions were done following the standard procedure of FAO (2006). Soil samples were collected from horizon of every soil profile 
following the quantitative sampling procedure of Schlichting et al. (1995). Samples were immediately brought to the screenhouse of the Department of Agronomy and Soil Science, VSU, Baybay City, Leyte for processing. Except those for bulk density determination, all soil samples were air-dried, pulverized using a wooden hammer and sieved in a 2-mm wire mesh to get the fine earth for the determination of most soil physical and chemical properties.

\section{Soil Physical and Chemical Analysis}

The collected undisturbed bulk samples were analyzed for bulk density by the paraffin clod method (Blake and Hartge, 1986). Porosity was calculated from the determined bulk density value and a constant particle density of $2.65 \mathrm{~g} / \mathrm{cm}^{3}$. Particle size distribution was determined by the pipette method (ISRIC, 1995). An ultrasonic disintegrator (Hielscher UP100H) was used to completely disperse the soil separates after addition of sodium hexametaphosphate as dispersing agent. Plasticity Index (PI) was calculated using the following pedotransfer equations provided by NSSC (1995): $\mathrm{PI}=$ clay- 21 ( $35-55 \%$ clay) and $\mathrm{PI}=$ clay- 15 ( $>55 \%$ clay).

The soil samples were also analyzed for $\mathrm{pH}_{\mathrm{H} 20}$ and $\mathrm{pH}_{\mathrm{Kсl}}$ potentiometrically using a soil-solution ratio of 1:2.5 (ISRIC, 1995). The numerical difference in the values of $\mathrm{pH}$ measured in $\mathrm{KCl}$ and $\mathrm{H}_{2} \mathrm{O}$ was obtained to get the delta $\mathrm{pH}(\Delta \mathrm{pH})$ (Mekaru and Uehara, 1972). Soil organic matter was analyzed following the Modified Walkley-Black method (Nelson and Sommers, 1982). Extraction of available P was done according to the Olsen method (Olsen and Sommers, 1982) and absorbance was read using spectrophotometer (Spectronic $20 \mathrm{D}^{+}$) at $880 \mathrm{~nm}$. Analysis of total $\mathrm{N}$ was done according to the micro-Kjeldahl method (ISRIC, 1995). Exchangeable bases were extracted by $1 \mathrm{~N} \mathrm{NH}_{4} \mathrm{OAc}(\mathrm{pH} 7.0)$ method according to ISRIC (1995). The quantification of exchangeable bases ( $\mathrm{K}, \mathrm{Ca}, \mathrm{Mg}, \mathrm{Na}$ ) was achieved with the use of atomic absorption spectrophotometer (Varian Spectra 220 FS). Potential cation exchange capacity (CEC) was determined using the ammonium acetate $\left(\mathrm{NH}_{4} \mathrm{OAc}\right)$ method at $\mathrm{pH} 7.0$ (ISRIC, 1995). Exchangeable acidity $\left(\mathrm{Al}^{3+}\right.$ and $\left.\mathrm{H}^{+}\right)$was analyzed using $1 \mathrm{~N} \mathrm{KCl}$ as extractant and quantified by titrating the extract with $0.1 \mathrm{~N} \mathrm{NaOH}$ (Thomas, 1982). Effective cation exchange capacity was calculated by summing up the amount of the exchangeable bases $(\mathrm{K}, \mathrm{Mg}, \mathrm{Ca}$, and $\mathrm{Na})$ and total acidity $\left(\mathrm{Al}^{3+}\right.$ and $\mathrm{H}^{+}$). The base saturation was calculated by dividing the sum of $\mathrm{K}, \mathrm{Mg}, \mathrm{Ca}$, and $\mathrm{Na}$ (bases) in $\mathrm{cmol}_{\mathrm{c}} \mathrm{kg}^{-1}$ soil by the potential CEC and multiplying the result by 100 . 


\section{RESULTS AND DISCUSSION}

\section{Site and Soil Characteristics}

\section{Soil Profile 1}

This soil is located in Lourdes, Abuyog on a footslope position at an elevation of $45 \mathrm{~m}$ above sea level (asl). The soil which developed from shale is well-drained and is covered with a mixture of native plant species (ferns and grasses) and crops such as coconuts and bananas (Table 1).

Soil profile 1 is poorly developed as indicated by the Ah-Bw-BC-CB horizon sequence (Fig. 3). Soil color ranges from dark brown (10YR 3/3) in the surface horizon to yellowish brown (10YR 5/4) in the subsurface horizon. Texture is silt loam ( $38.7 \%$ clay) in the upper portion of the profile which changes to silty clay ( $42.8 \%$ clay) in the lower part. The high amount of clay of this relatively young soil could be due to inheritance from the shale parent material. Structure is granular in the Ah horizon and fine subangular blocky in the B horizon. It is very porous as reflected by the low bulk density and high porosity values (Tables $2 \& 3$ ).

In terms of soil chemical properties, soil profile 1 is medium acid with an average $\mathrm{pH}_{\mathrm{H} 20}$ and negative delta $\mathrm{pH}$ values indicating negative net charge of the soil colloids (Mekaru and Uehara, 1972). Potential CEC of the soil is $33.53 \mathrm{cmol}_{\mathrm{c}} \mathrm{kg}^{-1}$ soil. Organic matter and total $\mathrm{N}$ contents decrease from the surface horizon to the subsurface horizons. Available $P$ is very low $\left(<1 \mathrm{mg} \mathrm{kg}^{-1}\right)$ throughout the soil profile while the exchangeable bases content is high ( $\mathrm{K}=0.37, \mathrm{Ca}=12.70, \mathrm{Mg}=12.56, \mathrm{Na}=0.28$, all in $\left.\mathrm{cmol}_{\mathrm{c}} \mathrm{kg}^{-1}\right)$ resulting in a base saturation of $72.3 \%$ (Table 4 ).

\section{Soil Profile 2}

This soil is located in an agricultural land planted with coconuts, bananas, fruit trees and some other field crops in Hilusig, Mahaplag (Fig. 3). The soil was derived from shale on a summit position at an elevation of $175 \mathrm{~m}$ asl. The site is nearly level and well-drained (Table 1 ).

From its morphology, this soil is moderately developed as indicated by the presence of an argillic B horizon (or Bt horizon). Soil color ranges from very dark brown (10YR 2/2) to dark yellowish brown (10YR 4/6) while texture varies from sandy clay loam ( $34.6 \%$ clay) to sandy clay ( $42.9 \%$ clay) from the surface to the subsurface. As in the case of soil profile 1 , 
Table 1. Site characteristics of the mountain soils in the Abuyog-Mahaplag-Baybay area in Leyte

\begin{tabular}{|c|c|c|c|c|c|c|c|c|}
\hline \multirow{2}{*}{ Site Characteristics } & \multicolumn{8}{|c|}{ Site } \\
\hline & 1 & 2 & 3 & 4 & 5 & 6 & 7 & 8 \\
\hline Location & $\begin{array}{l}\text { Lourdes, } \\
\text { Abuyog }\end{array}$ & $\begin{array}{l}\text { Upper Hilusig, } \\
\text { Mahaplag }\end{array}$ & $\begin{array}{l}\text { Lower Hilusig, } \\
\text { Mahaplag }\end{array}$ & $\begin{array}{l}\text { Cuatro de } \\
\text { Agosto, } \\
\text { Mahaplag }\end{array}$ & $\begin{array}{l}\text { Villa } \\
\text { Solidaridad, } \\
\text { Baybay }\end{array}$ & Mailhi, Baybay & $\begin{array}{c}\text { Kambongan, } \\
\text { Baybay }\end{array}$ & $\begin{array}{c}\text { Imelda, Makinhas, } \\
\text { Baybay }\end{array}$ \\
\hline \multirow[t]{2}{*}{ Coordinates } & $\mathrm{N} 10^{\circ} 40^{\prime} 13.3^{\prime \prime}$ & N10³9'51.8" & N10 $39^{\prime} 14.9^{\prime \prime}$ & 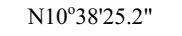 & N10 $37^{\circ} 36.3^{\prime \prime}$ & N10 $37^{\circ} 33.5^{\prime \prime}$ & $\mathrm{N} 10^{\circ} 37^{\prime} 49.7^{\prime \prime}$ & N10 $38^{\prime} 83.4^{\prime \prime}$ \\
\hline & 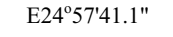 & 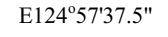 & 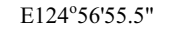 & $\mathrm{E} 124^{\circ} 56^{\prime} 28.1^{\prime \prime}$ & $\mathrm{E} 124^{\circ} 55^{\prime} 22.3^{\prime \prime}$ & E124ㅇ5' $55^{\prime} 18.7^{\prime \prime}$ & $\mathrm{E} 124^{\circ} 54^{\prime} 46.5^{\prime \prime}$ & $\mathrm{E} 124^{\circ} 51^{\prime} 37.1^{\prime \prime}$ \\
\hline Elevation & $45 \mathrm{~m}$ asl & $175 \mathrm{~m}$ asl & $163 \mathrm{~m}$ asl & $166 \mathrm{~m}$ asl & $357 \mathrm{~m}$ asl & $325 \mathrm{~m}$ as 1 & $327 \mathrm{~m}$ asl & $98 \mathrm{~m}$ asl \\
\hline Landform & $\begin{array}{l}\text { Sedimentary } \\
\text { mountain }\end{array}$ & $\begin{array}{l}\text { Sedimentary } \\
\text { mountain }\end{array}$ & $\begin{array}{l}\text { Sedimenatry } \\
\text { mountain }\end{array}$ & $\begin{array}{l}\text { Volcanic } \\
\text { mountain }\end{array}$ & $\begin{array}{l}\text { Volcanic } \\
\text { mountain }\end{array}$ & $\begin{array}{l}\text { Sedimentary } \\
\text { mountain }\end{array}$ & $\begin{array}{l}\text { Volcanic } \\
\text { mountain }\end{array}$ & Volcanic mountain \\
\hline Slope Position & Footslope & Summit & Backslope & Footslope & Summit & Shoulder & Shoulder & Summit \\
\hline Slope Gradient & Nearly level & Nearly level & Nearly level & Sloping & Gently sloping & Gently sloping & Gently sloping & Gently sloping \\
\hline Parent Material & Shale & Shale & Shale & Andesite & Andesite & Shale & Andesite & Andesite \\
\hline $\begin{array}{l}\text { Soil Moisture } \\
\text { Regime }\end{array}$ & Udic & Udic & Udic & Udic & Udic & Udic & Udic & Udic \\
\hline Soil Temperature & Isohyperthermic & Isohyperthermic & Isohyperthermic & Isohyperthermic & Isohyperthermic & Isohyperthermic & Isohyperthermic & Isohyperthermic \\
\hline Erosion & No evidence & No evidence & No evidence & Slight & No evidence & No evidence & No evidence & No evidence \\
\hline $\begin{array}{l}\text { Rock outcrops/ } \\
\text { stoniness }\end{array}$ & Few & Common & Many & Common & Very few & Many & Few & Very few \\
\hline Drainage & Well-drained & $\begin{array}{c}\text { Moderately } \\
\text { well-drained }\end{array}$ & Well-drained & $\begin{array}{l}\text { Moderately well- } \\
\text { drained }\end{array}$ & Well-drained & well-drained & Well-drained & Well-drained \\
\hline Land-use & Agricultural & Agricultural & Agricultural & $\begin{array}{l}\text { Agricultural } \\
\text { (Abandoned } \\
\text { Upland Farm) }\end{array}$ & $\begin{array}{c}\text { Agricultural } \\
\text { (Abandoned } \\
\text { Kaingin) }\end{array}$ & Agricultural & Agricultural & Agricultural \\
\hline Vegetation & $\begin{array}{c}\text { Mani-mani, } \\
\text { ferns, coconut, } \\
\text { banana, carabao } \\
\text { grass }\end{array}$ & $\begin{array}{c}\text { Coconut, } \\
\text { lanzones, } \\
\text { banana, ferns, } \\
\text { gabi, makahiya, } \\
\text { cogon grass }\end{array}$ & $\begin{array}{c}\text { Coconut, } \\
\text { banana, cogon } \\
\text { grass }\end{array}$ & $\begin{array}{l}\text { Cogon grass, } \\
\text { coconut, santol, } \\
\text { narra, cassava, } \\
\text { native lassiandra, } \\
\text { ferns }\end{array}$ & $\begin{array}{l}\text { Gmelina trees, } \\
\text { carabao grass, } \\
\text { fern, native } \\
\text { lassiandra, white } \\
\text { lantanas, } \\
\text { Christmas bush }\end{array}$ & $\begin{array}{c}\text { Coconut, } \\
\text { banana, carabao } \\
\text { grass }\end{array}$ & $\begin{array}{l}\text { Coconut, } \\
\text { gmelina trees }\end{array}$ & $\begin{array}{l}\text { Coconut, ferns, } \\
\text { carabao grass }\end{array}$ \\
\hline
\end{tabular}




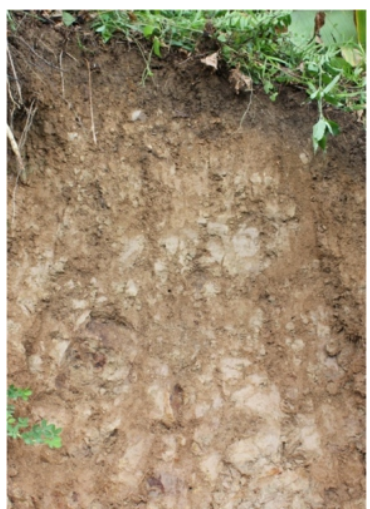

Soil Profile 1

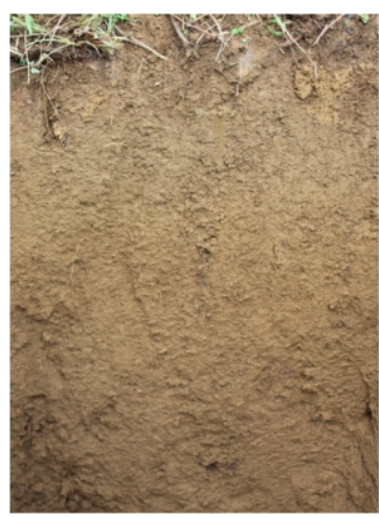

Soil Profile 5

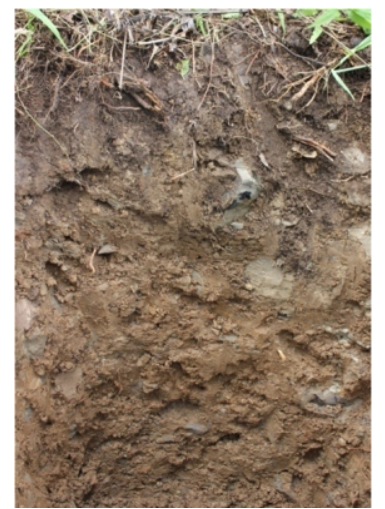

Soil Profile 2

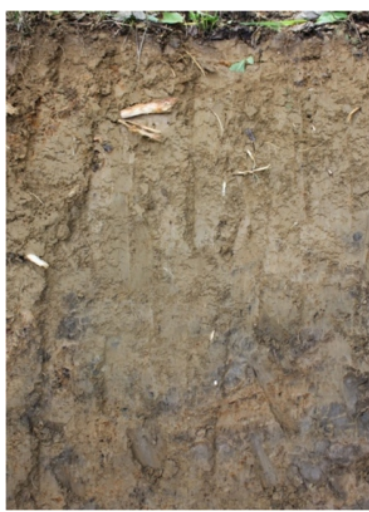

Soil Profile 6

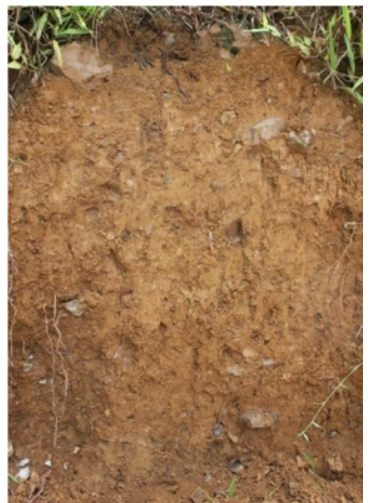

Soil Profile 3

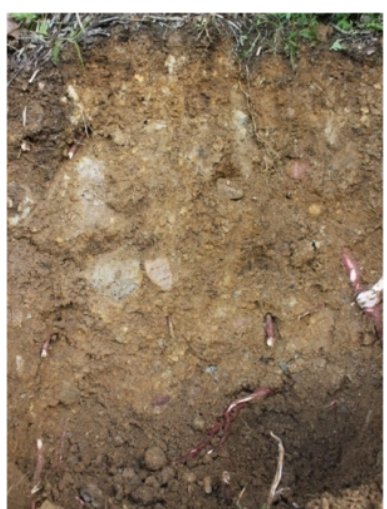

Soil Profile 7

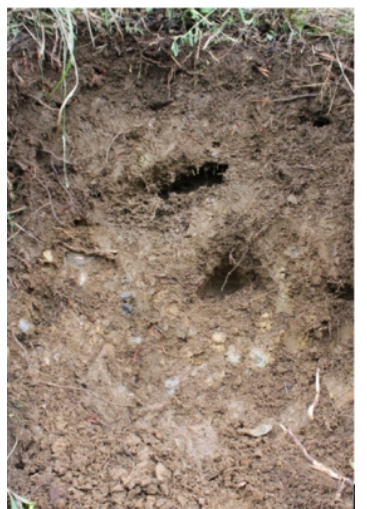

Soil Profile 4

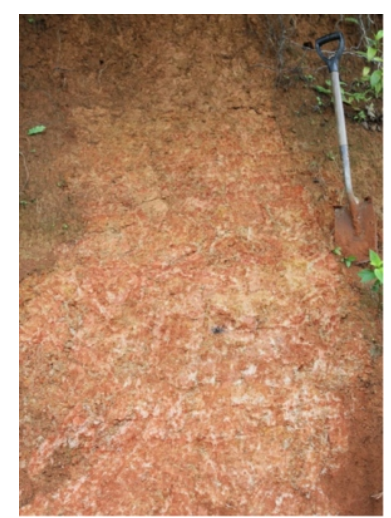

Soil Profile 8

Figure 3. Soil profiles of the mountain soils in the Abuyog-Mahaplag-Baybay area in Leyte that were studied 
inheritance of clay from the shale parent material largely explains the high clay content of this soil. It has sub-angular blocky structure and slightly plastic and sticky wet consistence. The soil is porous as shown by its low bulk density $\left(1.18 \mathrm{~g} \mathrm{~cm}^{-3}\right)$ and high porosity (56\%) (Tables $2 \& 3$ ).

In terms of soil chemical properties, the soil has $\mathrm{pH}_{\text {н20 }}$ slightly above 7.0 (neutral). The soil colloids have net negative charge as reflected by the negative delta $\mathrm{pH}$ values. Organic matter content decreases from $4.93 \%$ in the top horizon to $0.71 \%$ in the lowest horizon. Total $\mathrm{N}$ content is low having an average value of $0.11 \%$ while available $\mathrm{P}$ in the soil is moderate $\left(18 \mathrm{mg} \mathrm{kg}^{-1}\right)$. Potential CEC is moderately high varying from $57.71 \mathrm{cmol}_{c} \mathrm{~kg}^{-1}$ soil in the surface layer to $41.16 \mathrm{cmol}_{\mathrm{c}} \mathrm{kg}^{-1}$ in the subsurface. Exchangeable bases content of the soil is high $(\mathrm{K}=1.37, \mathrm{Ca}=30.70, \mathrm{Mg}=5.20, \mathrm{Na}=0.30$, all in $\mathrm{cmol}_{\mathrm{c}} \mathrm{kg}^{-1}$ ) resulting in a base saturation of $81.4 \%$ (Table 4 ).

\section{Soil Profile 3}

This well-developed soil has a horizon sequence of Ah-Bw-Bt-BC and is located at an elevation of $163 \mathrm{~m}$ ASL on the lower backslope of the mountain where soil profile 2 is also found. Soil profile 3 was also developed from shale. Land use of the area is agricultural and vegetation consists of coconut, banana and cogon (Table 1 ).

The soil has a surface horizon that is dark brown (10 YR 4/3), sandy clay loam (29\% clay) texture and granular structure, the latter imparts its friable consistence. In contrast, the subsurface horizons (B horizons) are yellowish red (10YR 5/8-4/6), sandy clay (38-42\% clay) and has subangular blocky structure making it slightly firm in consistence. The soil is generally porous having an average bulk density and porosity of $1.21 \mathrm{~g} \mathrm{~cm}^{-3}$ and 54\%, respectively (Table $2 \& 3$; Fig. 3).

Regarding soil chemistry,, the soil has an average $\mathrm{pH}_{\mathrm{н} 20}$ of 5.7, organic matter content of $1.44 \%$, total $\mathrm{N}$ of $0.07 \%$, available $\mathrm{P}$ of $1.60 \mathrm{mg} \mathrm{kg}^{-1}$, and exchangeable bases of 0.41 for $\mathrm{K}, 12.90$ for $\mathrm{Ca}, 5.30$ for $\mathrm{Mg}$ and 0.35 for $\mathrm{Na}$ (all in $\mathrm{cmol}_{\mathrm{c}} \mathrm{kg}^{-1}$ ). The negative charge of the soil colloids can be inferred from the negative delta $\mathrm{pH}$ values. Potential CEC of the soil is $31.50 \mathrm{cmol}_{c} \mathrm{~kg}$ ${ }^{1}$ soil while its base saturation is $60 \%$ (Table 4). A related soil developed from shale in the southwestern part of the island was found to have a clay fraction dominated by smectite, kaolinite and halloysite clay minerals (Navarrete et al., 2011). 
Table 2. Morphological characteristics of mountain soils in the Abuyog-Mahaplag-Baybay area in Leyte

\begin{tabular}{|c|c|c|c|c|c|c|c|c|c|c|}
\hline \multirow{2}{*}{$\begin{array}{c}\text { Soil } \\
\text { Profile/ } \\
\text { Horizon }^{\mathrm{A}}\end{array}$} & \multirow[b]{2}{*}{ Depth (cm) } & \multirow[b]{2}{*}{ Soil Color } & \multirow[b]{2}{*}{ Texture $^{\mathrm{B}}$} & \multirow[b]{2}{*}{ Structure $^{\mathrm{C}}$} & \multicolumn{2}{|c|}{ Consistence } & \multirow[b]{2}{*}{ Roots $^{\mathrm{F}}$} & \multirow[b]{2}{*}{ Pores $^{\mathrm{G}}$} & \multirow[b]{2}{*}{ Boundary ${ }^{\mathrm{H}}$} & \multirow{2}{*}{$\begin{array}{c}\text { Rock }^{\mathrm{I}} \\
\text { Fragments }\end{array}$} \\
\hline & & & & & Moist $^{D}$ & $\mathrm{Wet}^{\mathrm{E}}$ & & & & \\
\hline \multicolumn{11}{|c|}{ Soil Profile 1 (Lourdes, Abuyog) } \\
\hline $\mathrm{Ah}$ & $0-18$ & 10YR 3/3 (dark brown) & $\mathrm{SiCL}$ & $1 \mathrm{fsg}$ & fi & sst \& spl & $\mathrm{ff}$ & $\mathrm{ff}$ & $\mathrm{cs}$ & $\mathrm{n}$ \\
\hline $\mathrm{Bw}$ & $18-44$ & 10YR 4/4 (dark yellowish brown) & $\mathrm{SiCL}$ & $1 \mathrm{fsbk}$ & vfi & sst \& spl & vff & vff & cs & $\mathrm{n}$ \\
\hline $\mathrm{BC}$ & $44-70$ & 10YR 5/4 (yellowish brown) & $\mathrm{SiC}$ & $2 \mathrm{fsbk}$ & vfi & sst \& spl & vff & vff & $\mathrm{cs}$ & $\mathrm{m}$ \\
\hline CB1 & $70-95$ & 10YR 5/4 (yellowish brown) & $\mathrm{SiC}$ & $2 \mathrm{fsbk}$ & vfi & sst \& spl & vff & vff & ds & $\mathrm{m}$ \\
\hline CB2 & $95-128$ & 10YR 5/4 (yellowish brown) & $\mathrm{SiC}$ & $2 \mathrm{fsbk}$ & vfi & sst \& spl & $\mathrm{n}$ & $\mathrm{n}$ & ds & $\mathrm{m}$ \\
\hline \multicolumn{11}{|c|}{ Soil Profile 2 (Upper Hilusig, Mahaplag) } \\
\hline Ah1 & $0-13$ & 10YR 2/2 (very dark brown) & SCL & $1 \mathrm{fsbk}$ & fi & sst \& spl & $\mathrm{mf}$ & $\mathrm{fm}$ & cs & $\mathrm{f}$ \\
\hline Ah2 & $13-31$ & 10YR 4/2 (dark grayish brown) & SCL & $2 \mathrm{fsbk}$ & fi & sst \& spl & $\mathrm{cf}$ & vff & as & $\mathrm{m}$ \\
\hline Bt1 & $31-56$ & 10YR 4/4 (dark yellowish brown) & $\mathrm{SC}$ & $2 \mathrm{msbk}$ & vfi & st \& pl & vfvf & vff & as & $\mathrm{m}$ \\
\hline $\mathrm{Bt} 2$ & $56-78$ & 10YR 4/6 (dark yellowish brown) & $\mathrm{SC}$ & $2 \mathrm{msbk}$ & vfi & st \& pl & vfvf & vfvf & cs & $\mathrm{m}$ \\
\hline \multicolumn{11}{|c|}{ Soil Profile 3 (Lower Hilusig, Mahaplag) } \\
\hline $\mathrm{Ah}$ & $0-17$ & 10YR 4/3 (dark brown) & SCL & $1 \mathrm{fsg}$ & $\mathrm{Fr}$ & nst \& npl & $\mathrm{mm}$ & $\mathrm{cf}$ & cs & $\mathrm{m}$ \\
\hline $\mathrm{Bw}$ & $17-37$ & 5YR 5/8 (yellowish red) & $\mathrm{SC}$ & $1 \mathrm{fsbk}$ & $\mathrm{Fi}$ & sst \& spl & $\mathrm{fm}$ & $\mathrm{ff}$ & $\mathrm{cs}$ & $\mathrm{m}$ \\
\hline Bt1 & $37-70$ & 5YR 4/6 (yellowish red) & $\mathrm{SC}$ & $2 \mathrm{msbk}$ & $\mathrm{Fi}$ & St \& pl & $\mathrm{ff}$ & vff & $\mathrm{cs}$ & $\mathrm{c}$ \\
\hline Bt2 & $70-95$ & 5YR 4/6 (yellowish red) & $\mathrm{SC}$ & $2 \mathrm{msbk}$ & $\mathrm{Fi}$ & St \& pl & vfvf & vff & $\mathrm{cs}$ & $\mathrm{c}$ \\
\hline $\mathrm{BC}$ & $95-112$ & 5YR 4/6 (yellowish red) & $\mathrm{SC}$ & $2 \mathrm{msbk}$ & $\mathrm{Fi}$ & St \& pl & vfvf & vff & ds & $\mathrm{c}$ \\
\hline \multicolumn{11}{|c|}{ Soil Profile 4 (Cuatro de Agosto, Mahaplag) } \\
\hline Ap & $0-16$ & $\begin{array}{l}10 \mathrm{YR} 3 / 2 \text { (very dark grayish } \\
\text { brown) }\end{array}$ & $\mathrm{SiCL}$ & $2 \mathrm{fsbk}$ & fr & sst \& spl & $\mathrm{cf}$ & $\mathrm{cf}$ & cs & $\mathrm{n}$ \\
\hline Bwl & $16-32$ & 10YR 4/3 (brown) & $\mathrm{SiC}$ & $3 \mathrm{msbk}$ & fi & sst \& spl & $\mathrm{cf}$ & $\mathrm{cf}$ & cs & $\mathrm{c}$ \\
\hline Bw2 & $32-54$ & 10YR 4/4 (dark yellowish brown) & $\mathrm{SiC}$ & $2 \mathrm{msbk}$ & fi & st \& pl & vfvf & vfvf & $\mathrm{cw}$ & $\mathrm{c}$ \\
\hline $\mathrm{BC}$ & $54-82$ & 10YR 5/4 (yellowish brown) & $\mathrm{SiC}$ & $2 \mathrm{msbk}$ & fi & sst \& spl & vfvf & vfvf & $\mathrm{cW}$ & $\mathrm{c}$ \\
\hline
\end{tabular}

${ }^{\mathrm{A}}$ According to IUSS Working Group WRB (2006)

${ }^{\text {в }}$ SC, Sandy Clay; SCL, Sandy Clay Loam; SiCL, Silty Clay Loam; SiL, Silt Loam; SiC, Silty Clay; C, Clay

${ }^{\mathrm{c}}$ 1, weak; 2 , moderate; 3 , strong; f, fine; $\mathrm{m}$, medium; sbk, sub-angular blocky; g, granular

${ }^{\mathrm{D}}$ fi, firm; vfi, very firm; fr, friable 
Soil Profile 5 (Villa Solidaridad, Baybay)

$\begin{array}{lcl}\text { Ap1 } & 0-8 & \begin{array}{l}\text { 10YR 3/2 (very dark grayish } \\ \text { brown) }\end{array} \\ \text { Ap2 } & 8-16 & \text { 10YR 4/3 (brown) } \\ \text { Bw1 } & 16-36 & \text { 10YR 4/4 (dark yellowish brown) } \\ \text { Bw2 } & 36-55 & \text { 10YR 4/6 (dark yellowish brown) } \\ \text { Bw3 } & 55-77 & \text { 10YR 4/6 (dark yellowish brown) }\end{array}$

$\begin{array}{cc}\mathrm{SiCL} & 1 \mathrm{fg} \\ \mathrm{SiCL} & 1 \mathrm{fg} \\ \mathrm{SiC} & 2 \mathrm{msbk} \\ \mathrm{SiC} & 3 \mathrm{msbk} \\ \mathrm{SiC} & 3 \mathrm{msbk}\end{array}$

$\begin{array}{|cc|}\text { sst \& spl } & \text { cm } \\ \text { sst \& spl } & \text { ff } \\ \text { st \& pl } & \text { vfvf } \\ \text { st \& pl } & \text { vfvf } \\ \text { st \& pl } & \text { vfvf }\end{array}$

Soil Profile 6 (Mailhi, Baybay)

$\begin{array}{ccl}\mathrm{Ah} & 0-17 & \text { 10YR 5/4 (yellowish brown) } \\ \mathrm{Bw} & 17-33 & \text { 10YR 5/4 (yellowish brown) } \\ \mathrm{BC} 1 & 33-52 & 10 \mathrm{YR} \mathrm{5/3} \mathrm{(brown)} \\ \mathrm{BC} 2 & 52-78 & 10 \mathrm{YR} \mathrm{4/3} \mathrm{(brown)}\end{array}$

$\begin{array}{cc}\mathrm{SiCL} & 1 \mathrm{fsbk} \\ \mathrm{SiC} & 2 \mathrm{fsbk} \\ \mathrm{SiCL} & 2 \mathrm{msbk} \\ \mathrm{SiCL} & 2 \mathrm{msbk}\end{array}$

Soil Profile 7 (Kambongan, Baybay)

$\begin{array}{ccl}\text { Ah } & 0-16 & \text { 10YR 4/6 (dark yellowish brown) } \\ \text { Bw } & 16-32 & \text { 10YR 4/6 (dark yellowish brown) } \\ \text { BC1 } & 32-50 & \text { 10YR 4/6 (dark yellowish brown) } \\ \text { BC2 } & 50-71 & \text { 10YR 5/8 (yellowish brown) }\end{array}$

$\begin{array}{ll}\mathrm{SiCL} & 1 \mathrm{fsbk} \\ \mathrm{SiC} & 1 \mathrm{fsbk} \\ \mathrm{SiL} & 1 \mathrm{msbk} \\ \mathrm{SiL} & 1 \mathrm{msbk}\end{array}$

$\begin{array}{cll}\text { sfi } & \text { sst \& spl } & \text { ff } \\ \text { fi } & \text { sst \& spl } & \text { ff } \\ \text { fi } & \text { sst \& spl } & \text { vff } \\ \text { fi } & \text { sst \& spl } & \text { vff }\end{array}$

ff
ff
vff
vff

Soil Profile 8 (Makinhas, Baybay)

\begin{tabular}{|c|c|c|c|c|c|c|c|c|c|c|}
\hline $\mathrm{Ah}$ & $0-20$ & 5YR 4/4 (reddish brown) & $\mathrm{SiC}$ & $3 \mathrm{fSbk}$ & fr & st \& pl & $\mathrm{cm}$ & $\mathrm{cm}$ & $\mathrm{dw}$ & $\mathrm{f}$ \\
\hline Bt1 & $20-43$ & 5YR 5/6 (yellowish red) & $\mathrm{C}$ & $3 \mathrm{fSbk}$ & fr & vst \& pl & $\mathrm{cm}$ & $\mathrm{cm}$ & $\mathrm{dw}$ & $\mathrm{f}$ \\
\hline Bt2 & $43-66$ & 5YR 5/6 (yellowish red) & $\mathrm{C}$ & $2 \mathrm{msbk}$ & fr & vst \& pl & $\mathrm{ff}$ & $\mathrm{cm}$ & $\mathrm{ds}$ & $\mathrm{f}$ \\
\hline Bt3 & $66-94$ & 5YR 5/8 (yellowish red) & $\mathrm{C}$ & $2 \mathrm{msbk}$ & fr & vst \& pl & $\mathrm{ff}$ & $\mathrm{ff}$ & $\mathrm{cs}$ & $\mathrm{f}$ \\
\hline Bt4 & $94-126$ & 5YR 5/8 (yellowish red) & $\mathrm{C}$ & $3 \mathrm{msbk}$ & fr & st \& pl & vff & $\mathrm{ff}$ & $\mathrm{ds}$ & $\mathrm{c}$ \\
\hline $\mathrm{BC} 1$ & $126-154$ & $2.5 \mathrm{YR} 4 / 8$ (red) & $\mathrm{SiC}$ & $3 \mathrm{msbk}$ & $\mathrm{fr}$ & st \& pl & vff & vff & ds & $\mathrm{c}$ \\
\hline $\mathrm{BC} 2$ & $154-178$ & 2.5 YR $4 / 8$ (red) & $\mathrm{SiC}$ & $3 \mathrm{msbk}$ & fr & st \& pl & vff & vfvf & $\mathrm{ds}$ & $\mathrm{c}$ \\
\hline $\mathrm{BC} 3$ & $178-214$ & 2.5 YR $4 / 8$ (red) & $\mathrm{SiC}$ & $1 \mathrm{fsbk}$ & fr & st \& pl & $\mathrm{n}$ & vfvf & $\mathrm{dw}$ & $\mathrm{c}$ \\
\hline BC4 & 214 below & 2.5 YR $4 / 8$ (red) & $\mathrm{SiC}$ & $1 \mathrm{fsbk}$ & fr & st \& pl & $\mathrm{n}$ & vfvf & $\mathrm{dw}$ & $\mathrm{c}$ \\
\hline
\end{tabular}

${ }^{\mathrm{E}}$ nst, not sticky; sst, slightly sticky; st, sticky; vst, very sticky

$\mathrm{n}$, none; vf, very few very fine; vff, very few fine; fm, few medium; cf, common fine; cm, common medium; mm, many medium ${ }^{\mathrm{H}}$ a, abrupt; c, clear; d, diffuse; s, smooth; w, wavy

${ }^{\mathrm{I}} \mathrm{n}$, none; f, few; c, common; m, many 
Table 3. Physical characteristics of mountain soils in the Abuyog-Mahaplag-Baybay area in Leyte

\begin{tabular}{|c|c|c|c|c|c|c|c|c|}
\hline \multirow[t]{2}{*}{$\begin{array}{l}\text { Soil Profile/ } \\
\text { Horizon }{ }^{\mathrm{A}}\end{array}$} & \multirow[t]{2}{*}{ Depth $(\mathrm{cm})$} & \multicolumn{3}{|c|}{$\begin{array}{c}\text { Particle } \\
\text { Size } \\
\text { Distribution }\end{array}$} & \multirow[t]{2}{*}{ Textural Class } & \multirow[t]{2}{*}{$\begin{array}{c}\text { Bulk } \\
\text { Density } \\
\left(\mathrm{g} / \mathrm{cm}^{3}\right)\end{array}$} & \multirow[t]{2}{*}{$\begin{array}{c}\text { Porosity } \\
(\%)\end{array}$} & \multirow[t]{2}{*}{$\begin{array}{l}\text { Plasticity } \\
\text { Index* }\end{array}$} \\
\hline & & Sand & $\begin{array}{l}\text { Silt } \\
(\%) \\
\end{array}$ & Clay & & & & \\
\hline & & & & & Soil Profile 1 (Lourdes, Abuyog) & & & \\
\hline $\mathrm{Ah}$ & $0-18$ & 6.72 & 55.12 & 38.16 & Silty clay loam & 1.12 & 57.74 & 17.16 \\
\hline $\mathrm{Bw}$ & $18-44$ & 5.48 & 55.21 & 39.31 & Silty clay loam & 1.16 & 56.23 & 18.31 \\
\hline $\mathrm{BC}$ & $44-70$ & 3.68 & 53.45 & 42.87 & silty clay & 1.20 & 54.72 & 21.87 \\
\hline CB1 & $70-95$ & 3.52 & 53.56 & 42.92 & silty clay & 1.20 & 54.72 & 21.92 \\
\hline CB2 & $95-128$ & 3.37 & 54.03 & 42.60 & silty clay & 1.25 & 52.83 & 21.60 \\
\hline & & & & & Soil Profile 2 (Upper Hilusig, Mahaplag 1) & & & \\
\hline Ah1 & $0-13$ & 39.80 & 25.64 & 34.56 & Sandy clay loam & 1.10 & 58.87 & 13.56 \\
\hline Ah2 & $13-31$ & 34.81 & 37.56 & 27.63 & Sandy clay loam & 1.16 & 56.23 & n.d \\
\hline Bt1 & $31-56$ & 44.67 & 12.98 & 42.35 & Sandy clay & 1.23 & 55.47 & 21.35 \\
\hline $\mathrm{Bt} 2$ & $56-78$ & 44.15 & 12.96 & 42.89 & Sandy clay & 1.25 & 53.58 & 21.89 \\
\hline & & & & & Soil Profile 3 (Lower Hilusig, Mahaplag 2) & & & \\
\hline $\mathrm{Ah}$ & $0-17$ & 49.78 & 21.23 & 28.99 & Sandy clay loam & 1.15 & 56.60 & n.d \\
\hline $\mathrm{Bw}$ & $17-37$ & 50.23 & 11.52 & 38.25 & Sandy clay & 1.20 & 54.72 & 17.25 \\
\hline Bt1 & $37-70$ & 48.42 & 11.23 & 40.35 & Sandy clay & 1.18 & 55.47 & 19.35 \\
\hline Bt2 & $70-95$ & 46.87 & 11.24 & 41.89 & Sandy clay & 1.24 & 53.21 & 20.89 \\
\hline $\mathrm{BC}$ & $95-112$ & 47.11 & 11.35 & 41.54 & Sandy clay & 1.28 & 52.08 & 20.54 \\
\hline & & & & & Soil Profile 4 (Cuatro de Agosto, Mahaplag) & & & \\
\hline Ap & $0-16$ & 9.66 & 55.25 & 35.09 & Silty clay loam & 1.00 & 62.26 & 14.09 \\
\hline Bw1 & $16-32$ & 8.20 & 50.67 & 41.13 & Silty clay & 1.03 & 61.13 & 20.13 \\
\hline Bw2 & $32-54$ & 6.25 & 51.80 & 41.95 & Silty clay & 1.09 & 58.87 & 20.95 \\
\hline $\mathrm{BC}$ & $54-82$ & 6.10 & 51.90 & 42.00 & Silty clay & 1.13 & 57.36 & 21.00 \\
\hline
\end{tabular}




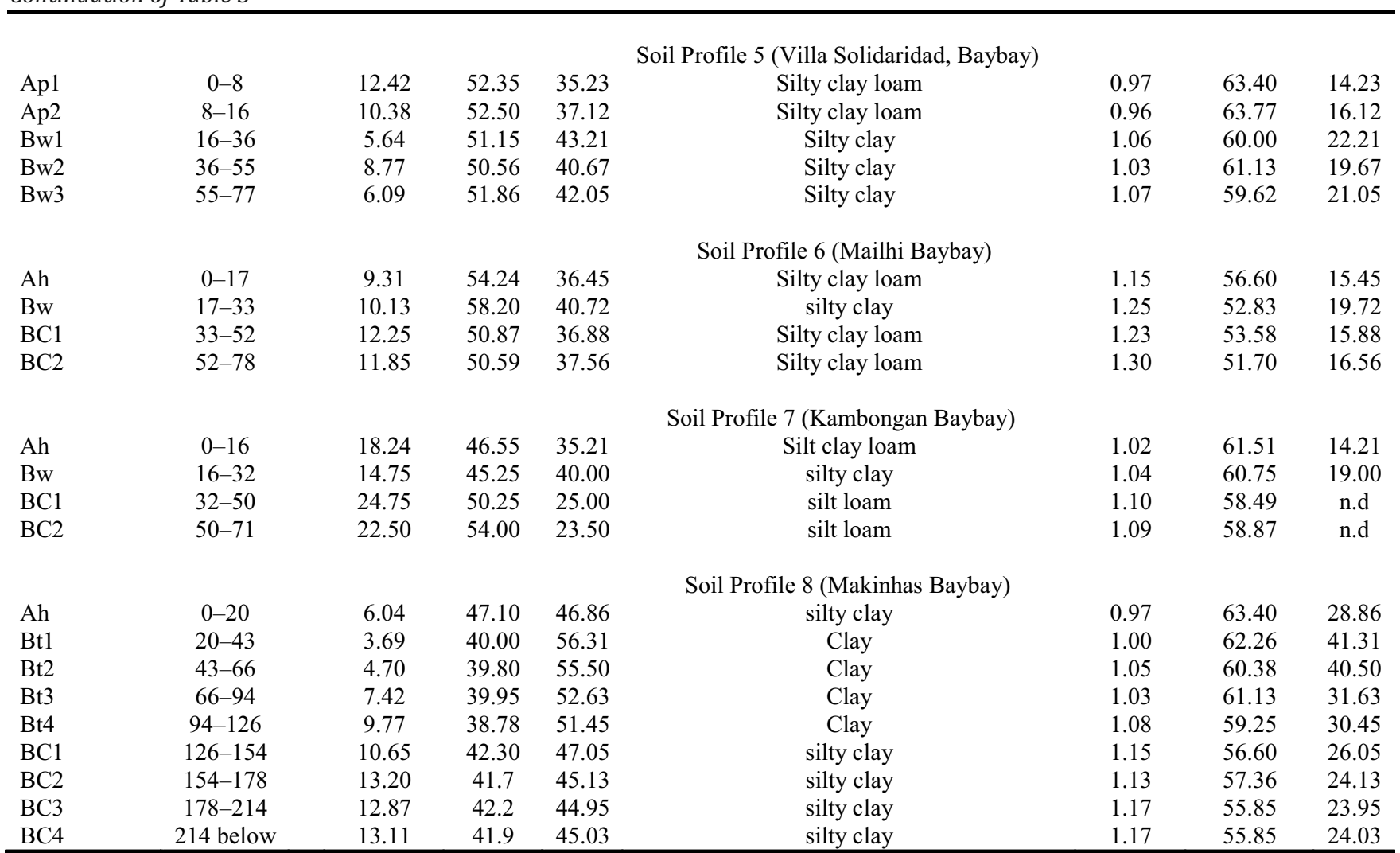


Table 4. Chemical characteristics of mountain soils in the Abuyog-Mahaplag-Baybay area in Leyte

\begin{tabular}{|c|c|c|c|c|c|c|c|c|c|c|c|}
\hline \multirow{2}{*}{$\begin{array}{l}\text { Soil } \\
\text { Profile/ } \\
\text { Horizon }^{\text {A }}\end{array}$} & \multirow[t]{2}{*}{$\begin{array}{l}\text { Depth } \\
(\mathrm{cm})\end{array}$} & \multicolumn{2}{|c|}{$\mathrm{pH}(1: 2.5)$} & & \multirow{2}{*}{$\begin{array}{l}\text { OM } \\
(\%) \\
\end{array}$} & \multirow{2}{*}{$\begin{array}{c}\text { Total } \\
\mathrm{N} \\
(\%) \\
\end{array}$} & \multirow{2}{*}{$\begin{array}{c}\text { Available } \\
\text { P } \\
(\mathrm{mg} / \mathrm{kg})\end{array}$} & \multicolumn{4}{|c|}{$\begin{array}{l}\text { Exchangeable Bases }\left(\mathrm{cmol}_{\mathrm{c}}\right. \\
/ \mathrm{kg} \text { soil) }\end{array}$} \\
\hline & & $\mathrm{KCl}$ & $\mathrm{H}_{2} \mathrm{O}$ & & & & & $\mathrm{K}$ & $\mathrm{Ca}$ & $\mathrm{Mg}$ & $\mathrm{Na}$ \\
\hline \multicolumn{12}{|c|}{ Soil Profile 1 (Lourdes, Abuyog) } \\
\hline $\mathrm{Ah}$ & $0-18$ & 3.99 & 5.31 & -1.32 & 3.12 & 0.19 & 0.26 & 0.42 & 15.97 & 13.78 & 0.23 \\
\hline $\mathrm{Bw}$ & $18-44$ & 3.60 & 5.34 & -1.74 & 0.78 & 0.06 & 0.24 & 0.31 & 11.55 & 11.79 & 0.26 \\
\hline $\mathrm{BC}$ & $44-70$ & 3.61 & 5.40 & -1.79 & 0.39 & 0.05 & 0.26 & 0.39 & 10.77 & 12.05 & 0.34 \\
\hline CB1 & $70-95$ & 3.61 & 5.37 & -1.76 & 0.58 & 0.03 & 0.26 & n.d & n.d & n.d & n.d \\
\hline CB2 & $95-128$ & 3.65 & 4.77 & -1.12 & 0.78 & 0.03 & 0.09 & n.d & n.d & n.d & n.d \\
\hline \multicolumn{12}{|c|}{ Soil Profile 2 (Upper Hilusig, Mahaplag ) } \\
\hline Ah1 & $0-13$ & 5.97 & 7.22 & -1.25 & 4.93 & 0.26 & 14.86 & 1.89 & 35.42 & 6.86 & 0.33 \\
\hline Ah2 & $13-31$ & 5.94 & 7.24 & -1.30 & 2.58 & 0.10 & 19.42 & 1.67 & 32.39 & 4.93 & 0.26 \\
\hline Bt1 & $31-56$ & 5.55 & 7.11 & -1.56 & 1.04 & 0.06 & 23.43 & 2.26 & 27.85 & 4.15 & 0.27 \\
\hline Bt2 & $56-78$ & 5.35 & 7.23 & -1.88 & 0.71 & 0.04 & 12.57 & 1.11 & 27.19 & 4.97 & 0.36 \\
\hline \multicolumn{12}{|c|}{ Soil Profile 3 (Lower Hilusig, Mahaplag ) } \\
\hline $\mathrm{Ah}$ & $0-17$ & 4.28 & 5.79 & -1.51 & 2.71 & 0.13 & 1.87 & 0.86 & 14.70 & 6.50 & 0.29 \\
\hline $\mathrm{Bw}$ & $17-37$ & 4.22 & 6.03 & -1.81 & 1.24 & 0.08 & 0.98 & 0.24 & 14.36 & 4.57 & 0.47 \\
\hline Bt1 & $37-70$ & 3.81 & 5.77 & -1.96 & 1.17 & 0.06 & 0.98 & 0.18 & 11.79 & 4.47 & 0.34 \\
\hline $\mathrm{Bt} 2$ & $70-95$ & 3.74 & 5.53 & -1.79 & 1.11 & 0.05 & 1.42 & 0.18 & 11.80 & 5.02 & 0.32 \\
\hline $\mathrm{BC}$ & $95-112$ & 3.89 & 5.52 & -1.63 & 0.98 & 0.05 & 2.63 & 0.18 & 11.80 & 5.86 & 0.33 \\
\hline \multicolumn{12}{|c|}{ Soil Profile 4 (Cuatro de Agosto, Mahaplag) } \\
\hline Ap & $0-16$ & 3.80 & 5.29 & -1.49 & 3.74 & 0.20 & 0.15 & 0.18 & 2.02 & 2.36 & 0.47 \\
\hline Bw1 & $16-32$ & 3.71 & 5.68 & -1.97 & 2.17 & 0.11 & 0.15 & 0.06 & 1.69 & 1.64 & 0.68 \\
\hline Bw2 & $32-54$ & 3.64 & 5.67 & -2.03 & 1.57 & 0.06 & 0.06 & 0.05 & 1.33 & 1.16 & 0.52 \\
\hline $\mathrm{BC}$ & $54-82$ & 3.58 & 5.66 & -2.08 & 0.98 & 0.05 & 0.06 & 0.02 & 1.19 & 0.97 & 0.31 \\
\hline \multicolumn{12}{|c|}{ Soil Profile 5 (Villa Solidaridad, Baybay) } \\
\hline Ap1 & $0-8$ & 3.74 & 4.77 & -1.03 & 4.65 & 0.30 & 0.80 & 0.17 & 0.59 & 0.30 & 0.18 \\
\hline Ap2 & $8-16$ & 3.85 & 4.65 & -0.80 & 4.30 & 0.19 & 0.77 & 0.10 & 0.42 & 0.11 & 0.16 \\
\hline Bw1 & $16-36$ & 3.80 & 4.97 & -1.17 & 2.04 & 0.02 & 0.21 & 0.03 & 0.31 & 0.04 & 0.21 \\
\hline Bw2 & $36-55$ & 3.73 & 4.95 & -1.22 & 1.51 & 0.05 & 0.11 & 0.02 & 0.32 & 0.03 & 0.23 \\
\hline Bw3 & $55-77$ & 3.73 & 4.40 & -1.67 & 1.24 & 0.05 & 0.15 & n.d & n.d & n.d & n.d \\
\hline \multicolumn{12}{|c|}{ Soil Profile 6 (Mailhi, Baybay) } \\
\hline $\mathrm{Ah}$ & $0-17$ & 3.46 & 4.94 & -1.48 & 1.31 & 0.11 & 0.24 & 0.32 & 5.40 & 14.01 & 0.43 \\
\hline $\mathrm{Bw}$ & $17-33$ & 3.51 & 5.44 & -1.93 & 0.65 & 0.14 & 0.09 & 0.10 & 6.34 & 16.70 & 0.58 \\
\hline $\mathrm{BC} 1$ & $33-52$ & 3.48 & 5.19 & -1.71 & 0.71 & 0.04 & 0.06 & 0.10 & 8.52 & 17.70 & 0.63 \\
\hline $\mathrm{BC} 2$ & $52-78$ & 3.41 & 5.50 & -2.09 & 0.45 & 0.03 & 0.47 & n.d & n.d & n.d & n.d \\
\hline \multicolumn{12}{|c|}{ Soil Profile 7 (Kambongan, Baybay) } \\
\hline $\mathrm{Ah}$ & $0-16$ & 3.86 & 5.17 & -1.31 & 2.85 & 0.14 & 2.17 & 0.33 & 5.04 & 2.24 & 0.88 \\
\hline Bw & $16-32$ & 3.75 & 5.19 & -1.44 & 1.51 & 0.07 & 0.48 & 0.23 & 4.18 & 1.58 & 0.93 \\
\hline $\mathrm{BC} 1$ & $32-50$ & 3.79 & 5.20 & -1.41 & 1.37 & 0.07 & 0.39 & 0.23 & 4.32 & 1.52 & 0.68 \\
\hline $\mathrm{BC} 2$ & $50-71$ & 3.68 & 5.08 & -1.40 & 0.78 & 0.05 & 0.47 & n.d & n.d & n.d & n.d \\
\hline
\end{tabular}

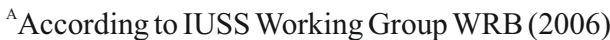

$\mathrm{n} . \mathrm{d}=$ not determined 
Soil Profile 8 (Makinhas, Baybay)

\begin{tabular}{lccccccccccc} 
Ah & $0-20$ & 3.46 & 4.70 & -1.24 & 3.53 & 0.17 & 0.45 & 0.12 & 1.66 & 1.36 & 0.17 \\
Bt1 & $20-43$ & 3.42 & 4.77 & -1.35 & 2.31 & 0.11 & 0.45 & 0.10 & 0.80 & 0.74 & 0.23 \\
Bt2 & $43-66$ & 3.43 & 4.82 & -1.39 & 1.70 & 0.08 & 0.42 & 0.09 & 0.66 & 0.70 & 0.22 \\
Bt3 & $66-94$ & 3.44 & 4.81 & -1.37 & 1.31 & 0.07 & 0.42 & 0.15 & 0.60 & 1.20 & 0.20 \\
Bt4 & $94-126$ & 3.43 & 4.82 & -1.39 & 0.78 & 0.04 & 0.39 & n.d & n.d & n.d & n.d \\
BC1 & $126-154$ & 3.40 & 4.73 & -1.33 & 0.52 & 0.04 & 0.30 & n.d & n.d & n.d & n.d \\
BC2 & $154-178$ & 3.35 & 4.82 & -1.47 & 0.26 & 0.04 & 0.25 & n.d & n.d & n.d & n.d \\
BC3 & $178-214$ & 3.33 & 4.89 & -1.56 & 0.32 & 0.03 & 0.31 & n.d & n.d & n.d & n.d \\
BC4 & 214 below & 3.34 & 4.92 & -1.58 & 0.26 & 0.03 & 0.28 & n.d & n.d & n.d & n.d \\
\hline
\end{tabular}

Continuation of Table 4

\begin{tabular}{|c|c|c|c|c|c|c|c|}
\hline \multirow{2}{*}{$\begin{array}{l}300 \\
\text { Profile/ } \\
\text { Horizon }\end{array}$} & \multirow[t]{2}{*}{$\begin{array}{c}\text { Depth } \\
\text { (cm) }\end{array}$} & \multicolumn{2}{|c|}{$\begin{array}{l}\text { Exchangeable } \\
\left(\mathrm{cmol}_{\mathrm{c}} / \mathrm{kg} \text { soil }\right)\end{array}$} & \multirow{2}{*}{$\begin{array}{l}\text { Exchangeable } \\
\text { Acidity } \\
\text { ( } \mathrm{cmol}_{\mathrm{c}} / \mathrm{kg} \text { soil) }\end{array}$} & \multicolumn{2}{|c|}{$\begin{array}{c}\mathrm{CEC} \\
\left(\mathrm{cmol}_{\mathrm{c}} / \mathrm{kg} \text { soil }\right)\end{array}$} & \multirow{2}{*}{$\begin{array}{c}\text { Base } \\
\text { Saturation } \\
(\%)\end{array}$} \\
\hline & & $\mathrm{Al}^{+3}$ & $\mathrm{H}^{+}$ & & Eff & Pot & \\
\hline \multicolumn{8}{|c|}{ Soil Profile 1 (Lourdes Abuyog) } \\
\hline $\mathrm{Ah}$ & $0-18$ & n.d & 0.10 & n.d. & n.d. & 38.12 & 79.74 \\
\hline $\mathrm{Bw}$ & $18-44$ & n.d. & 0.16 & n.d. & n.d. & 34.79 & 68.72 \\
\hline $\mathrm{BC}$ & $44-70$ & n.d. & 0.16 & n.d. & n.d. & 34.40 & 68.47 \\
\hline CB1 & $70-95$ & n.d. & 0.10 & n.d. & n.d & 35.75 & n.d \\
\hline CB2 & $95-128$ & n.d. & 0.10 & n.d. & n.d & 34.59 & n.d \\
\hline \multicolumn{8}{|c|}{ Soil Profile 2 (Upper Hilusig Mahaplag ) } \\
\hline Ah1 & $0-13$ & 0.05 & 0.05 & 0.10 & 44.60 & 57.71 & 77.10 \\
\hline Ah2 & $13-31$ & 0.05 & 0.05 & 0.10 & 39.35 & 46.99 & 83.52 \\
\hline $\mathrm{Bw}$ & $31-56$ & 0.05 & 0.05 & 0.10 & 34.65 & 41.56 & 83.12 \\
\hline Bt & $56-78$ & 0.05 & 0.05 & 0.10 & 33.73 & 41.16 & 81.70 \\
\hline \multicolumn{8}{|c|}{ Soil Profile 3 (Lower Hilusig Mahaplag ) } \\
\hline $\mathrm{Ah}$ & $0-17$ & 0.58 & 0.10 & 0.68 & 23.03 & 32.49 & 68.80 \\
\hline Bw & $17-37$ & 0.31 & 0.10 & 0.42 & 20.06 & 32.67 & 60.12 \\
\hline Bt1 & $37-70$ & 1.53 & 0.16 & 1.68 & 18.47 & 30.73 & 54.62 \\
\hline Bt2 & $70-95$ & 1.52 & 0.16 & 1.68 & 18.99 & 30.92 & 56.01 \\
\hline $\mathrm{BC}$ & $95-112$ & 0.68 & 0.10 & 0.78 & 18.96 & 30.92 & 58.77 \\
\hline \multicolumn{8}{|c|}{ Soil Profile 4 (Cuatro de Agosto Mahaplag) } \\
\hline Ap & $0-16$ & 0.89 & 0.26 & 1.15 & 6.18 & 13.35 & 37.66 \\
\hline Bw1 & $16-32$ & 1.37 & 0.47 & 1.84 & 5.91 & 13.15 & 30.95 \\
\hline Bw2 & $32-54$ & 1.90 & 0.47 & 2.37 & 5.43 & 12.76 & 23.98 \\
\hline $\mathrm{BC}$ & $54-82$ & 2.55 & 0.69 & 3.24 & 5.74 & 12.95 & 19.34 \\
\hline \multicolumn{8}{|c|}{ Soil Profile 5 (Villa Solidaridad Baybay) } \\
\hline Ap1 & $0-8$ & 0.94 & 0.83 & 1.77 & 3.01 & 9.49 & 13.02 \\
\hline Ap2 & $8-16$ & 1.41 & 0.52 & 1.93 & 2.72 & 9.10 & 8.70 \\
\hline Bw1 & $16-36$ & 1.72 & 0.31 & 2.03 & 2.63 & 8.69 & 6.89 \\
\hline Bw2 & $36-55$ & 1.31 & 0.30 & 1.62 & 2.22 & 7.54 & 8.04 \\
\hline Bw3 & $55-77$ & 1.61 & 0.16 & 1.77 & n.d & 7.54 & n.d \\
\hline
\end{tabular}




\begin{tabular}{|c|c|c|c|c|c|c|c|}
\hline \multicolumn{8}{|c|}{ Soil Profile 6 (Mailhi Baybay) } \\
\hline $\mathrm{Ah}$ & $0-17$ & 4.78 & 1.05 & 5.83 & 26.00 & 33.65 & 59.94 \\
\hline Bw & $17-33$ & 2.45 & 0.83 & 3.29 & 26.99 & 34.04 & 69.64 \\
\hline BC1 & $33-52$ & 1.63 & 0.79 & 2.41 & 29.37 & 34.59 & 77.93 \\
\hline $\mathrm{BC} 2$ & $52-78$ & 1.78 & 0.94 & 2.72 & n.d & 34.39 & n.d \\
\hline \multicolumn{8}{|c|}{ Soil Profile 7 (Kambongan Baybay) } \\
\hline $\mathrm{Ah}$ & $0-16$ & 0.48 & 0.11 & 0.59 & 9.09 & 19.15 & 44.39 \\
\hline Bw & $16-32$ & 1.11 & 0.32 & 1.43 & 8.36 & 18.75 & 36.94 \\
\hline BC1 & $32-50$ & 1.27 & 0.48 & 1.75 & 8.49 & 18.94 & 35.62 \\
\hline $\mathrm{BC} 2$ & $50-71$ & 1.37 & 0.55 & 1.92 & n.d & 17.78 & n.d \\
\hline \multicolumn{8}{|c|}{ Soil Profile 8 (Makinhas Baybay) } \\
\hline $\mathrm{Ah}$ & $0-20$ & 3.77 & 0.69 & 4.46 & 7.77 & 16.45 & 20.10 \\
\hline Bt1 & $20-43$ & 6.05 & 0.90 & 6.96 & 8.83 & 18.76 & 10.00 \\
\hline Bt2 & $43-66$ & 6.38 & 1.06 & 7.44 & 9.13 & 18.95 & 8.89 \\
\hline Bt3 & $66-94$ & 6.02 & 0.85 & 6.88 & 9.03 & 18.94 & 11.35 \\
\hline $\mathrm{Bt} 4$ & $94-126$ & 7.35 & 1.90 & 9.25 & n.d & 20.87 & n.d \\
\hline $\mathrm{BC} 1$ & $126-154$ & 8.38 & 1.92 & 10.31 & n.d & 19.32 & n.d \\
\hline $\mathrm{BC} 2$ & $154-178$ & 8.52 & 1.95 & 10.47 & n.d & 18.74 & n.d \\
\hline $\mathrm{BC} 3$ & $178-214$ & 8.53 & 1.68 & 10.22 & n.d & 18.94 & n.d \\
\hline $\mathrm{BC} 4$ & $\begin{array}{c}214 \\
\text { below }\end{array}$ & 8.53 & 1.85 & 10.38 & n.d & 17.97 & n.d \\
\hline
\end{tabular}

\footnotetext{
${ }^{\mathrm{A}}$ According to IUSS Working Group WRB (2006) n.d $=$ not determined
}

\section{Soil Profile 4}

The soil is located in Cuatro de Agosto, Mahaplag at an elevation of $166 \mathrm{~m}$ asl (Fig. 3). The area is an abandoned upland farm and is now dominated by a mixture of various crops and native plants. The soil profile examined is located on a moderately well-drained footslope position. It was derived from volcanic rocks particularly andesite (Table 1 ).

In terms of morpho-physical characteristics, soil profile 4 has a horizon sequence of Ap-Bw-BC. Soil color ranges from very dark grayish brown (10YR 3/2) in the top layer to yellowish brown $(10$ YR 5/4) at 54-82 cm depth. Texture is silty clay loam (35.0\% clay) in the A horizon which turns silty clay ( $41.8 \%$ clay) in all the lower horizons. Structure is sub-angular blocky throughout the soil profile; its consistence is friable on the surface but firm in the subsurface. The soil is very porous as shown by the bulk density values of $1.0 \mathrm{~g} \mathrm{~cm}^{-3}$ and porosity of $57-62 \%$ (Tables $2 \& 3$ ).

The soil has the following chemistry (profile average): $\mathrm{pH}_{\mathrm{H} 20}=5.6, \mathrm{OM}=$ $2.1 \%$, total $\mathrm{N}=0.10 \%$, available $\mathrm{P}=<1 \mathrm{mg} \mathrm{kg}^{-1}$, exchangeable bases of $0.07(\mathrm{~K})$, $1.56(\mathrm{Ca}), 1.53(\mathrm{Mg})$, and $0.50(\mathrm{Na})$ and exchangeable acidity of 2.15 (all in 
$\left.\mathrm{cmol}_{\mathrm{c}} \mathrm{kg}^{-1}\right)$. The soil has negative delta $\mathrm{pH}$ values indicating negative net charge of soil colloids. Potential CEC of the soil is low at $13.0 \mathrm{cmol}_{\mathrm{c}} \mathrm{kg}^{-1}$ soil. Its base saturation ranges from $38 \%$ in the surface horizon to $19 \%$ in the lower horizon (Table 4).

\section{Soil Profile 5}

This soil is located in Villa Solidaridad, Baybay at an elevation of $357 \mathrm{~m}$ asl, the highest site that was examined for this study. The area is an abandoned upland farm and now covered with trees, carabao grass, cogon grass, fern and other native plants. Dominant rock is volcanic particularly andesite (Table 1). The soil profile is located on a summit position (Fig. 3).

Soil color ranges from very dark grayish brown (10 YR 3/2) in the surface horizon to dark yellowish brown (10 YR 4/6) in the lower horizons. Texture ranges from silty clay loam ( $35.2 \%$ clay) to silty clay ( $42 \%$ clay) with depth. The soil is generally soft and friable throughout its profile which is also reflected by the low bulk density value of $<1.0 \mathrm{~g} \mathrm{~cm}^{-3}$ and more than $60 \%$ porosity in the top layer (Tables $2 \& 3$ ). These characteristics are typical for young volcanic soils (Shoji et al., 1993).

This weakly developed soil with a horizon sequence of $\mathrm{Ap}-\mathrm{Bw}$, is strongly acidic $\left(\mathrm{pH}_{\mathrm{H} 20}<5.0\right)$ and has the following nutrient status: $\mathrm{OM}=$ 4.65 to $1.24 \%$, total $\mathrm{N}=0.30-0.05 \%$, available $\mathrm{P}=<1 \mathrm{mg} \mathrm{kg}{ }^{-1}$, and exchangeable bases of $0.08(\mathrm{~K}), 0.41(\mathrm{Ca}), 0.12(\mathrm{Mg})$, and $0.20(\mathrm{Na})$ (all in $\mathrm{cmol}_{\mathrm{c}} \mathrm{kg}^{-1}$ ). Exchangeable acidity of the soil is considerable and ranges from 1.62 to $2.03 \mathrm{cmol}_{c} \mathrm{~kg}^{-1}$. Potential CEC and base saturation of the soil are low having values of $8.5 \mathrm{cmol}_{c} \mathrm{~kg}^{-1}$ soil and $9.20 \%$, respectively (Table 4).

Several physical and chemical characteristics of this soil indicate that it is closely related to the young volcanic soils (Andisols) in the Ormoc portion of the central highlands of Leyte that were the subject of previous pedological research (Asio, 1996; Navarrete et al., 2008). The clay fraction of this volcanic soil in Ormoc is composed primarily of gibbsite, allophane, imogolite, goethite including chlorite and vermiculite (Asio, 1996).

\section{Soil Profile 6}

This soil is found in a sedimentary hill in Mailhi, Baybay at an elevation of $325 \mathrm{~m}$ asl (Fig. 3). Land use of the site is agricultural and the dominant vegetation consists of coconut, banana, fruit trees and grasses. The soil profile examined is located on a gently sloping shoulder position. Parent 
rock of the soil is shale (Table 1 ).

In terms of morphological characteristics, the soil has an Ah-Bw-BC horizon sequence. Its color varies from yellowish brown (10YR 5/4) in the surface layer to brown (10YR 5/3) in the subsurface. The soil is generally silty clay loam (38\% clay), firm and has sub-angular blocky structure. Bulk density is low ranging from 1.15 to $1.30 \mathrm{~g} \mathrm{~kg}^{-1}$ from the upper to the lower part of the profile (Tables $2 \& 3$ ).

In terms of chemical characteristics, the soil is medium acidic $\mathrm{pH}_{\mathrm{H} 20}=$ 5.3), contains low organic matter $(0.78 \%)$, has a total $\mathrm{N}$ of $0.08 \%$ and available $\mathrm{P}$ of $0.21 \mathrm{mg} \mathrm{kg}^{-1}$. Exchangeable bases content is generally high particularly in the surface horizon as follows: $0.32(\mathrm{~K}), 5.40(\mathrm{Ca}), 14.01$ $\left(\mathrm{Mg}\right.$ ) and $0.43(\mathrm{Na})$ (all in $\mathrm{cmol}_{\mathrm{c}} \mathrm{kg}^{-1}$ ). Potential CEC of the soil is $34.2 \mathrm{cmol}_{\mathrm{c}}$ $\mathrm{kg}^{-1}$ soil and the base saturation is $69.0 \%$ (Table 4).

\section{Soil Profile 7}

The area where this soil occurs in Kambongan, Baybay is dominated by coconut and Gmelina trees. The soil profile examined is located in a welldrained and gently sloping shoulder position at an elevation of $327 \mathrm{~m}$ asl. Geology of the area is characterized by volcanic rocks particularly andesite (Table 1; Fig. 3).

The poorly developed soil has a horizon sequence of Ah-Bw-BC. It has a characteristic dark yellowish brown color (10YR 4/6) and has a silty clay loam (35\% clay) surface horizon which changes to silt loam (23\% clay) below. The soil is firm when moist and slightly sticky and plastic when wet. Bulk density values of the soil are close to $1.0 \mathrm{~g} \mathrm{~cm}^{-3}$ as well as a porosity of about $60 \%$ (Tables $2 \& 3$ ).

Regarding chemical properties, this soil is medium acid $\left(\mathrm{pH}_{\mathrm{H} 20}=5.2\right)$ and contains low $\mathrm{OM}(1.63 \%)$. Total $\mathrm{N}$ is $0.08 \%$, available $\mathrm{P}$ is $0.90 \mathrm{mg} \mathrm{kg}^{-1}$. It has moderate amounts of exchangeable bases $(\mathrm{K}=0.20, \mathrm{Ca}=4.50, \mathrm{Mg}=$ $1.75, \mathrm{Na}=0.83$, all in $\left.\mathrm{cmol}_{\mathrm{c}} \mathrm{kg}^{-1}\right)$. Potential CEC and base saturation of the soil are $18.7 \mathrm{cmol}_{\mathrm{c}} \mathrm{kg}^{-1}$ soil and $38.9 \%$, respectively (Table 4).

\section{Soil Profile 8}

This soil is the thick and red soil that can be observed along the highway in Imelda, Makinhas, Baybay at an elevation of $98 \mathrm{~m}$ asl (Fig. 3). Vegetation cover consists largely of coconut, trees, ferns and in open areas grasses are dominant. The soil which developed from andesite volcanic 
rock, is located on a well-drained summit position (Table 1). A microscopic examination of the sand fraction reveals the presence of ferromagnesian minerals (hornblende and pyroxene), feldspar and quartz which confirms the andesite origin of the soil (Fig. 4).

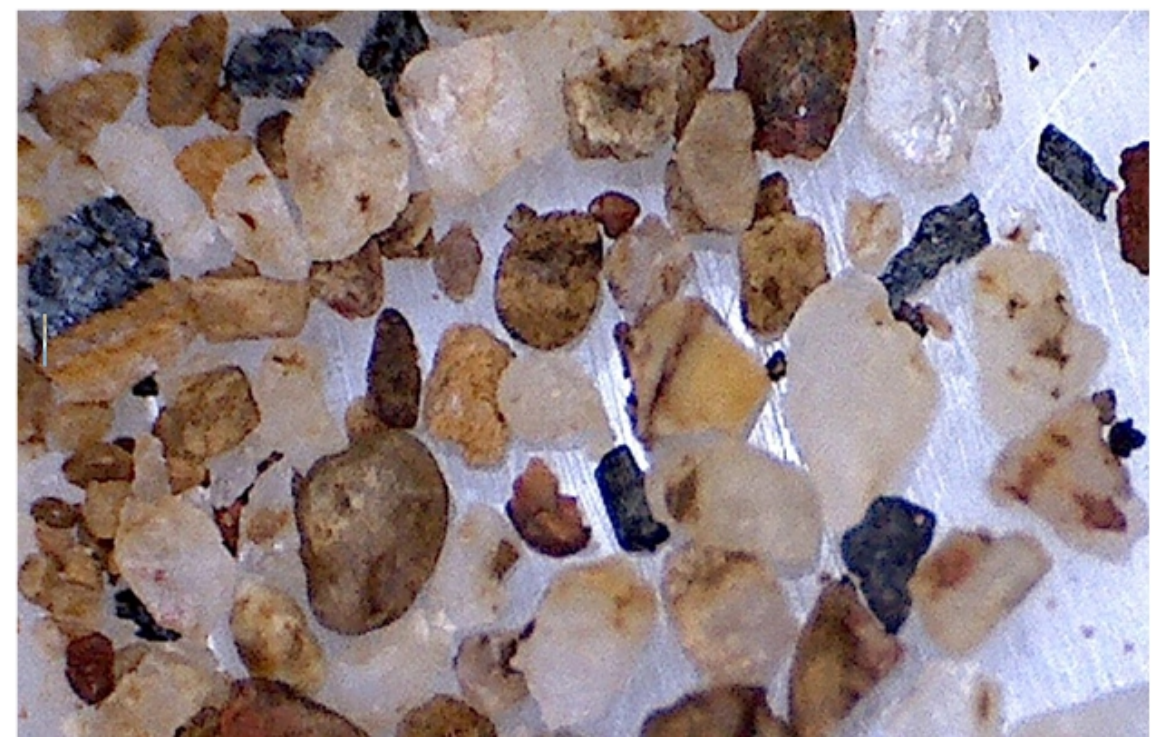

Figure 4 . A microscope view (100x) of the sand fraction of the highly weathered soil from andesite (Soil Profile 8) showing the presence of dark-colored ferromagnesian minerals (pyroxene and hornblende) and light colored minerals (feldspar and quartz)

The soil profile which is about 4 meters thick was examined from a relatively recent quarry. Horizon sequence is Ah-Bt-BC. Soil color of the upper $1.26 \mathrm{~m}$ is reddish brown (10YR 4/4) which changes to red (2.5YR $4 / 8$ ) below until a depth of more than $3 \mathrm{~m}$. Texture of the upper profile is clay ( $53 \%$ clay) which becomes silty clay ( $45 \%$ clay) in the lower part of the profile. The whole soil profile is generally friable when moist probably due to the strong aggregation imparted by the high iron oxide content (Sanchez, 1976) and is very plastic and very sticky. The very plastic characteristic of the soil is also shown by its plasticity index ranging from 24 to 40 (high plasticity). The bulk density values of the upper portion of the profile are close to $1.0 \mathrm{~g} \mathrm{~cm}^{-3}$ which increase in the lower part of the profile to $1.16 \mathrm{~g} \mathrm{~cm}^{-3}$. Consequently, the soil is very porous with total porosity values ranging from 55 to $63 \%$ (Tables $2 \& 3$ ). 


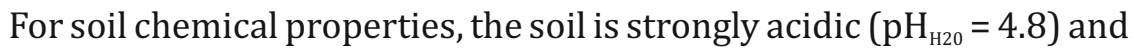
has negative delta $\mathrm{pH}$ values, both properties do not change considerably with depth. In contrast, $\mathrm{OM}$ abruptly decreases below the one meter depth. The soil has very low available $\mathrm{P}\left(<1 \mathrm{mg} \mathrm{kg}^{-1}\right)$ and low exchangeable bases $\left(\mathrm{K}=0.11, \mathrm{Ca}=0.93, \mathrm{Mg}=1.00\right.$ and $\left.\mathrm{Na}=0.20, \mathrm{cmol}_{\mathrm{c}} \mathrm{kg}^{-1}\right)$. Exchangeable acidity level of the soil is high which increases with depth. Potential CEC is low (18.8 $\mathrm{cmol}_{\mathrm{c}} \mathrm{kg}^{-1}$ soil) as well as its base saturation (12.6\%) indicating that the soil is highly weathered and infertile (Table 4). A comparable highly weathered soil in Mt. Pangasugan, Baybay, Leyte has a clay fraction that is dominated by kaolinite and halloysite silicate clay minerals including high amounts of the iron oxides goethite and hematite (Asio, 1996).

The apparent differences in some soil properties between the upper and lower portions of the soil profile suggest that the soil probably developed from two parent materials of closely similar chemical composition. It is also possible that the upper one meter of the soil profile is a recent formation from a younger volcanic deposit.

\section{Effects of Parent Material and Topography}

The soils in the study area vary in properties and stage of development primarily because of the effect of parent material and topography (specifically slope positions). Soil profile 1, which developed from shale on a footslope position, is relatively young (poorly developed) which can be explained by its unstable slope position which is a depositional surface. The high amount of clay of this young soil is probably inherited from the shale parent material. The soil is classified as Fluventric Eutropept in the Soil Taxonomy (Soil Survey Staff, 1999) or Eutric Fluvisol in the World Reference Base classification system (WRB, 1998) (Table 5). Soil profile 2 which formed from shale in a summit position, is well developed and has a thick solum, which can be explained by deeper weathering due to its relatively stable slope position (summit) that enhances vertical movement of water. It is classified as Typic Hapludalf (Soil Taxonomy) and as Haplic Luvisol (WRB). Soil profile 3, also from shale parent material, has a well developed soil and a thick solum located in a backslope position. It has a high base saturation all throughout due to the depositional process from the upper slope in which accumulation of organic matter as well as minerals is high. It is classified as Typic Hapludalf (Soil Taxonomy) or as Haplic Luvisol (WRB). Soil profile 4, which is situated at the footslope and which developed from andesite volcanic rock, is relatively young (poorly 
developed) and is classified as Typic Dystropept (Soil Taxonomy) or Dystric Cambisol (WRB). The classification name reflects the low nutrient status of the soil. Soil profiles 5 and 8 developed from andesite rocks at the summit position of a volcanic mountain. Loss of bases is indicated by the low values of base saturation and $\mathrm{pH}$. The moderate to high clay contents of both soils is probably the result of weathering of the andesite parent material. Although both are classified as Typic Kandiudult (Soil Taxonomy) or as Haplic Alisol (WRB), soil profile 8 is more weathered than soil profile 5. In fact, it is worth mentioning here that the classification name of soil profile 5 is not reflective of the morpho-physical properties of this soil as observed in the field. It appears more as a Hapludand or Haplic Andosol because of its high porosity, high friability and slight thixotropic property generally similar to the Andisols studied in other parts of the central highlands of Leyte (Asio, 1996; Zikeli et al., 1999; Navarrete et al., 2008). There is therefore a need for further evaluation of this soil profile. On the other hand, soil profile 8 is a typical Ultisol that is deep, clayey, red in color and acidic. Soil profiles 6 and 7 are located at the shoulder of a sedimentary hill and volcanic hill, respectively. Both soils are young, which could be attributed to their unstable slope position. In this position, surface flow of water is high which causes erosion and transport of soil and elements. The high amount of clay in soil profile 6 could have been inherited from its shale parent material rather than from neoformation of clay. In Soil Taxonomy, soil 6 is classified as a Typic Eutropept while soil profile 7 is a Typic Dystropept. In WRB, soil profile 6 is classified as a Eutric Cambisol while soil profile 7 is a Dystric Cambisol. The higher nutrient status of soil profile 6 is the effect of parent material.

\section{CONCLUSION}

Based on the results of the study, it can be concluded that: a) All soils studied varied in morpho-physical and chemical characteristics which partly reflect the parent material and slope position. b) Soils derived from shale (Soil profiles 1, 2, 3 and 6) developed into young (Fluventric Eutropepts) and well-developed (Typic Hapludalfs) soils which are generally high in base saturation. On the other hand, soils derived from andesite (Soil profiles 4, 5, 7 and 8) developed into young (Typic Dystropepts) and highly weathered soils (Typic Kandiudults) depending on the physiographic position. Regardless of parent material, soils on summit slope position tended to be better developed than soils on 
footslope and shoulder slope positions. The study showed that the influence of parent material on soil development was modified by the topographic position.

Table 5. Soil classification of studied soils based on Soil Taxonomy and World Reference Base

\begin{tabular}{|c|c|c|c|c|c|}
\hline Soil & $\begin{array}{c}\text { Parent } \\
\text { Material }\end{array}$ & Slope Position & $\begin{array}{c}\text { Development } \\
\text { Stage }\end{array}$ & $\begin{array}{c}\text { Soil Taxonomy } \\
\text { Classification }\end{array}$ & $\begin{array}{c}\text { WRB } \\
\text { Classification }\end{array}$ \\
\hline Soil Profile 1 & Shale & Footslope & $\begin{array}{l}\text { Poorly- } \\
\text { developed }\end{array}$ & $\begin{array}{l}\text { Fluventric } \\
\text { Eutropept }\end{array}$ & Eutric Fluvisol \\
\hline Soil Profile 2 & Shale & Summit & $\begin{array}{l}\text { Well- } \\
\text { developed }\end{array}$ & Typic Hapludalf & Haplic Luvisol \\
\hline Soil Profile 3 & Shale & Backslope & $\begin{array}{l}\text { Well- } \\
\text { developed }\end{array}$ & Typic Hapludalf & Haplic Luvisol \\
\hline Soil Profile 4 & Andesite & Footslope & $\begin{array}{l}\text { Poorly- } \\
\text { developed }\end{array}$ & Typic Dystropept & $\begin{array}{l}\text { Dystric } \\
\text { Cambisol }\end{array}$ \\
\hline Soil Profile 5 & Andesite & Summit & $\begin{array}{l}\text { Well- } \\
\text { developed }\end{array}$ & Typic Kandiudult & Haplic Alisol \\
\hline Soil Profile 6 & Shale & Shoulder & $\begin{array}{l}\text { Poorly- } \\
\text { developed }\end{array}$ & Typic Eutropept & $\begin{array}{l}\text { Eutric } \\
\text { Cambisol }\end{array}$ \\
\hline Soil Profile 7 & Andesite & Shoulder & $\begin{array}{l}\text { Poorly- } \\
\text { developed }\end{array}$ & Typic Dystropept & $\begin{array}{l}\text { Dystric } \\
\text { Cambisol }\end{array}$ \\
\hline Soil Profile 8 & Andesite & Summit & $\begin{array}{l}\text { Well- } \\
\text { developed }\end{array}$ & Typic Kandiudult & Haplic Alisol \\
\hline
\end{tabular}

\section{ACKNOWLEDGMENT}

The authors are grateful to the Department of Science and Technology (DOST) for awarding the first author (Ms. D.S. Maranguit) an Accelerated Science and Technology Human Resource Development Project (ASTHRDP)National Science Consortium graduate scholarship that made her MSc studies and graduate thesis (on which this paper was based) possible. The following members of her Graduate Advisory Committee are also thanked for their comments and suggestions on the thesis manuscript: Dr. Suzette B. Lina (Soil Science) and Dr. Ma. Juliet C. Ceniza (Tropical Ecology). 


\section{REFERENCES}

ASIO, V.B. 1996. Characteristics, weathering, formation, and degradation of soils from volcanic rocks in Leyte, Philippines. Hohenheimer Bodenkundliche Hefte Vol 33, Stuttgart, Germany.

ASIO, V.B., R. JAHN, K. STAHR, and J. MARGRAF. 1998. Soils of the tropical forests of Leyte, Philippines, II. Impact of different land uses on status of organic matter and nutrient availability In: Soils of Tropical Forest Ecosystems (A. Schulte \& D Ruhiyat, eds. Springer-Verlag Berlin. Pp: 37-44.

ASIO, V.B., C.C. CABUNOS JR., and Z.S. CHEN. 2006. Morphology, physiochemical characteristics, and fertility of soils from Quaternary limestone in Leyte, Philippines. Soil Science 171 (8): 648-661.

ASIO, V.B., R. JAHN, F.O. PEREZ, I.A. NAVARRETE, AND S.M. ABIT JR. 2009. A review of soil degradation in the Philippines. Annals of Tropical Research 31: 69-94.

AURELIO, M.A. 2000. Shear partitioning in the Philippines: constraints from Philippine fault and global positioning. Island Arc 9: 584-597.

BARRERA, A., I. ARISTORENAS, and J. TINGZON. 1954. Soil survey of Leyte Province, Philippines. Bureau of Print, Manila.

BLAKE, G.R. and K.H. HARTGE. 1986. Methods of soil analysis, Part 1. American Society of Agronomy-Soil Science Society of America, 677 South Segoe Road, Madison, WI 53711, USA.

DIMALANTA C.B., L.O. SUERTE, G.P. YUMUL, R.A.TAMAYO AND E.G.L. RAMOS. 2006. A Cretaceous supra-subduction oceanic basin source for Central Philippine ophiolitic basement complexes: geological and geophysical constraints. Geosciences Journal 10: 305-320.

FOOD AND AGRICULTURE ORGANIZATION OF THE UNITED NATIONS (FAO). 2006. Guidelines for soil description (4th edn). Rome, Italy.

HARTEMINK A.E. AND E.M. BRIDGES, 1995. The influence of parent material on soil fertility degradation in the coastal plain of Tanzania. Land Degradation and Rehabilitation 6: 215-221. 
Characteristics of mountain soils in central leyte

HUANG, W.T. 1962. Petrology. McGraw-Hill Book Company, New York.

INTERNATIONAL SOIL REFERENCE AND INFORMATION CENTER (ISRIC). 1995. Procedures for soil analysis (L.P. Van Reuwijk, Editor). Wageningen, The Netherlands.

JAPAN INTERNATIONAL COOPERATION AGENCY (JICA). 1990. Report on the mineral deposits and tectonics of two contrasting geologic environments in the Republic of the Philippines. Terminal Report. JICA and Metal Mining Agency of Japan.

MEKARU, T. and G. UEHARA. 1972. Anion adsorption in ferruginous tropical soils. Soil Sci. Soc. Amer. Proc. 36: 296-300

NATIONAL SOIL SURVEY CENTER (NSSC). 1995. Soil survey laboratory information manual. Soil Survey Information Report No. 45, Version 1.0, May 1995. Soil Survey Lab., Lincoln,Nebraska, NRCS-USDA.

NAVARRETE, I.A., K. TSUTSUKI, R. KONDO AND V.B. ASIO. 2008. Genesis of soils across a late Quaternary volcanic landscape in the humid tropical island of Leyte, Philippines. Australian Journal of Soil Research 46: 1-12.

NAVARRETE, I.A., K. TSUTSUKI, and R.A. NAVARRETE. 2010. Humus composition and the structural characteristics of humic substances in soils under different land uses in Leyte, Philippines. Soil Science and Plant Nutrition. 56: 289-296.

NAVARETTE, I.A., K. TSUTSUKI, V.B ASIO, M. TANI AND J. SUETA. 2011. Chemical, mineralogical and morphological characteristics of a Late Quaternary sedimentary-rock derived soils in Leyte, Philippines. Soil Science 176:699-708

NELSON, D.W. and L.E. SOMMERS. 1982. Methods of soil analysis, Part 2. American Society of Agronomy-Soil Science Society of America, 677 South Segoe Road, Madison, WI 53711, USA

OLSEN, S.R. and L.E. SOMMERS. 1982. Methods of soil analysis, Part 1. American Society of Agronomy-Soil Science Society of America, 677 South Segoe Road, Madison, WI 53711, USA 
RUHE, R.V. 1960. Elements of the soil landscape. Trans. $7^{\text {th }}$ Int. Congress Soil Science, Madison, Wisconsin 4: 165-170.

SANCHEZ, P.A. 1976. Properties and management of soils in the tropics. Wiley and Sons, New York.

SCHLICHTING, E., H.P. BLUME, and K. STAHR. 1995. Bodenkundliches Practikum (2nd edn.) Blackwell, Berlin.

SHOJI, S., M. NANZYO, and R.A DAHLGREN. 1993. Volcanic ash soils. Genesis, properties and utilization. Development in Soil Science 21, Elsevier, Armsterdam.

SOIL SURVEY STAFF, 1999. Soil Taxonomy: a basic system of soil classification for making and interpreting soil surveys. U.S. Dept of Agriculture, Washington, D.C.

THOMAS, G.W. 1982. Exchangeable cations.. In: Methods of soil analysis. Part 2. Chemical and microbiological properties ( $2^{\text {nd }}$ ed.) (A.L PAGE, Editor). Agron. Soc. Amer., Inc. and Soil Sci. Amer. Inc., Madison, Wisconsin. Pp:159-165.

WORLD REFERENCE BASE (WRB). 1998. World Reference Base for soil resources. World Soil Resources Report 84, ISRIC-FAO. Rome, Italy.

ZIKELI, S., V.B. ASIO and R. JAHN. 2000. Nutrient status of soils in the rain forest of Mt. Pangasugan, Leyte, Philippines. Annals of Tropical Research 22: 78-88. 